\title{
Article
}

\section{Self-Collection of Saliva Specimens as a Suitable Alternative to Nasopharyngeal Swabs for the Diagnosis of SARS-CoV-2 by RT-qPCR}

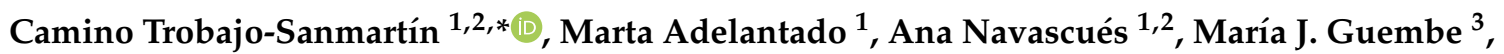 \\ Isabel Rodrigo-Rincón ${ }^{2,3,4} \mathbb{D}^{\text {, Jesús Castilla }}{ }^{2,5,6} \mathbb{C}^{-}$and Carmen Ezpeleta ${ }^{1,2}$ \\ 1 Department of Clinical Microbiology, Complejo Hospitalario de Navarra, 31008 Pamplona, Spain; \\ marta.adelantado.lacasa@navarra.es (M.A.); ana.navascues.ortega@navarra.es (A.N.); \\ carmen.ezpeleta.baquedano@navarra.es (C.E.) \\ 2 Instituto de Investigación Sanitaria de Navarra (IdiSNA), 31008 Pamplona, Spain; \\ mi.rodrigo.rincon@navarra.es (I.R.-R.); jcastilc@navarra.es (J.C.) \\ 3 Servicio de Apoyo a la Gestión Clínica y Continuidad Asistencial, Complejo Hospitalario de Navarra, \\ 31008 Pamplona, Spain; mguembes@navarra.es \\ 4 La Red de Investigación en Servicios de Salud en Enfermedades Crónicas (REDISSEC), 28029 Madrid, Spain \\ 5 Instituto de Salud Pública de Navarra, 31003 Pamplona, Spain \\ 6 CIBER Epidemiología y Salud Pública (CIBERESP), 28029 Madrid, Spain \\ * Correspondence: camino.trobajo.sanmartin@navarra.es
}

\section{check for}

updates

Citation: Trobajo-Sanmartín, C.; Adelantado, M.; Navascués, A.; Guembe, M.J.; Rodrigo-Rincón, I.; Castilla, J.; Ezpeleta, C.

Self-Collection of Saliva Specimens as a Suitable Alternative to Nasopharyngeal Swabs for the Diagnosis of SARS-CoV-2 by RT-qPCR. J. Clin. Med. 2021, 10, 299. https://doi.org/10.3390/jcm10020299

Received: 9 December 2020 Accepted: 12 January 2021 Published: 15 January 2021

Publisher's Note: MDPI stays neutral with regard to jurisdictional clai$\mathrm{ms}$ in published maps and institutional affiliations.

Copyright: (C) 2021 by the authors. Licensee MDPI, Basel, Switzerland. This article is an open access article distributed under the terms and conditions of the Creative Commons Attribution (CC BY) license (https:// creativecommons.org/licenses/by/ $4.0 /)$.

\begin{abstract}
A nasopharyngeal swab is a sample used for the diagnosis of SARS-CoV-2 infection. Saliva is a sample easier to obtain and the risk of contagion for the professional is lower. This study aimed to evaluate the utility of saliva for the diagnosis of SARS-CoV-2 infection. This prospective study involved 674 patients with suspected SARS-CoV-2 infection. Paired nasopharyngeal and saliva samples were processed by RT-qPCR. Sensitivity, specificity, and kappa coefficient were used to evaluate the results from both samples. We considered the influence of age, symptoms, chronic conditions, and sample processing with lysis buffer. Of the 674 patients, $636(94.4 \%)$ had valid results from both samples. The virus detection in saliva compared to a nasopharyngeal sample (gold standard) was 51.9\% (95\% CI: $46.3 \%-57.4 \%$ ) and increased to $91.6 \%$ (95\% CI: 86.7\%-96.5\%) when the cycle threshold $(\mathrm{Ct})$ was $\leq 30$. The specificity of the saliva sample was $99.1 \%(95 \% \mathrm{CI}$ : 97.0\%-99.8\%). The concordance between samples was 75\% ( $\kappa=0.50 ; 95 \% \mathrm{CI}: 0.45-0.56)$. The Ct values were significantly higher in saliva. In conclusion, saliva sample utility is limited for clinical diagnosis, but could be a useful alternative for the detection of SARS-CoV-2 in massive screening studies, when the availability of trained professionals for sampling or personal protection equipment is limited.
\end{abstract}

Keywords: saliva; nasopharyngeal; SARS-CoV-2; COVID-19; RT-qPCR

\section{Introduction}

A novel $\beta$-coronavirus Severe Acute Respiratory Syndrome Coronavirus 2 (SARSCoV-2) is the causative agent of the current coronavirus disease 2019 (COVID-19) pandemic that has affected the world since December 2019 [1,2]. The rapid spread of SARS-CoV-2, becoming a public health emergency, has forced the need for new diagnostics methods, vaccines, and therapies [3].

The World Health Organization (WHO) recommends the amplification of viral RNA by reverse transcription quantitative real-time polymerase chain reaction (RT-qPCR) from nasopharyngeal (NP) and oropharyngeal (OP) for the diagnosis and monitoring of infected patients for SARS-CoV-2 [4-6]. Nevertheless, the NP and OP specimen collection is considered an invasive procedure that may cause patient discomfort or light bleeding. 
Furthermore, this sample collection requires close contact between the health professional and the patient, which increases the risk of work-related transmission $[7,8]$.

Several studies have suggested saliva as a suitable sample for the detection of respiratory viruses, including the new, SARS-CoV-2 [7-9]. The collection and preservation of saliva samples are points of controversy in the different studies [10,11]. Furthermore, these studies present high variability in the methodology and the sample size.

Saliva collection could be easily performed by the patients themselves without the participation of a health care professional which reduces the risk of transmission and minimizes the use of material protection resources [7,9]. Therefore, this study aims to evaluate the utility of saliva samples for the diagnosis of SARS-CoV-2 infection and identify under what conditions a saliva sample could be useful.

\section{Experimental Section}

\subsection{Patients and Clinical Specimens}

From the 6th to the 12th of August 2020, a prospective study was performed among primary healthcare patients suspected of SARS-CoV-2 infection. Consecutive patients who came to an authorized place to collect samples for detection of SARS-CoV-2 were invited to participate in this study. All patients received an informative document with detailed information about the study objective and voluntary participation description. Those who voluntarily accept to participate received a sterile tube without conservation media and a written protocol for saliva suitable self-collection (Protocol S1). Nasopharyngeal swabs were collected by qualified nurses who had previously been trained. At the same time, the participants collected the saliva samples by themselves following Protocol S1. The nasopharyngeal sample was put in a sterile tube containing a viral transport medium. All samples were stored at room temperature until their transport to the clinical microbiology department of the Navarra Hospital Complex. Saliva samples were sent to the microbiology laboratory and frozen at $-80^{\circ} \mathrm{C}$ until processing.

Among all the pairs of samples received during the study period, the present study initially included all pairs with positive NP samples $(n=337)$ and 337 randomly selected pairs among those with negative NP samples (a total of 674 pairs of samples) to compare the results from NP and saliva samples.

\subsection{SARS-CoV-2 Detection by RT-PCR Assay}

Nasopharyngeal samples were processed with two different methods, 552 samples were extracted using the STARMag $96 \times 4$ universal extraction system (Seegene, Seoul, Korea) with the Hamilton Microlab STARlet automation robot (Hamilton Company, Reno, NV, USA) and then RT-qPCR was performed using the Allplex ${ }^{\mathrm{TM}}$ 2019-nCoV assay (Seegene, Seoul, Korea) on the CFX96 real-time PCR detection system (Bio-Rad, Hercules, CA, USA) following the manufacturer's instructions. The remaining 122 samples were analyzed on the Cobas ${ }^{\circledR} 6800$ platform (Roche Diagnostics GmbH, Mannheim, Germany) following the manufacturer's instructions. Although we used two different PCR kits (subject to laboratory and commercial stock availability), both methods detect the E SARS-CoV-2 gene.

All saliva samples were processed individually using the STARMag $96 \times 4$ universal extraction system and Allplex ${ }^{\mathrm{TM}}$ 2019-nCoV assay (Seegene, Seoul, Korea) for the RT-qPCR, following the manufacturer's instructions.

Saliva samples were collected in sterile tubes that did not contain conservation media, so we decided to see if buffer lysis's addition before processing influenced the RT-qPCR results. To evaluate the sample processing controlled over the RT-qPCR results obtained, some saliva samples were diluted on a lysis buffer (1:3) and inactivated for $10 \mathrm{~min}$ before RNA extraction.

\subsection{Statistical Analysis}

Sensitivity, specificity, and 95\% confidence intervals (CI) were calculated to assess patient diagnostic performance with valid result in saliva samples considering the virus's 
detection from an NP sample as the gold standard method. Alternatively, we evaluated saliva sensitivity for detection of infection among all patients, by including invalid results as non-positive samples. The kappa coefficient $(\kappa)$ was used to estimate the agreement between the saliva RT-qPCR and NP swab RT-qPCR results. Stratified analysis was conducted according to the cycle threshold $(\mathrm{Ct})$. For this purpose, two layers were considered: Ct greater and less than 30 because that cycle number is the one that the literature points out as the most relevant as an infective capacity proxy [12,13].

Sensitivity, specificity, and kappa coefficient were evaluated according to the sex, age ( $\leq 14$ and $>14$ years), chronic conditions, Ct for NP samples $(\leq 30$ and $>30)$, symptoms (yes/no), and saliva processing with lysis buffer (yes/no).

Means and standard deviations were calculated for quantitative variables and percentages for categorical variables. The associations between categorical variables were studied using a Chi-squared test or Fisher's exact test when necessary. Exact methods were used to obtain 95\% confidence intervals (CI) of proportions. The student's t-test for paired measures was used to compare $\mathrm{Ct}$ values between NP and saliva samples.

\subsection{Ethical Issues}

Samples were obtained with the verbal consent of participants. The Ethical Committee for Clinical Research of Navarra approved the study protocol, PI_2020/96.

\section{Results}

\subsection{Patients' Description}

Of 674 participants with paired samples tested, the median age was $36 \pm 19$ years old, 55.5\% were women, $103(15.3 \%)$ were children (0-14 years), and $571(84.7 \%)$ were aged $>14$ years. In terms of clinical presentation, $333(49.4 \%)$ patients presented symptoms compatible with COVID-19 disease, and 341 (50.6\%) subjects were asymptomatic. No significant differences were observed by sex, age, and chronic conditions between those participants with positive and negative results for SARS-CoV-2 in the NP sample or between those with $\mathrm{Ct} \leq 30$ and $\mathrm{Ct}>30$. However, a significantly higher proportion of people with symptoms was observed in patients with SARS-CoV-2 positive result in $\mathrm{NP}$ sample, and those with $\mathrm{Ct} \leq 30$. Among those NP samples which were tested using Cobas ${ }^{\circledR} 6800$ of Roche Diagnostics, a higher proportion of positive samples were detected but no differences were observed in Ct values (Table 1).

Table 1. Main characteristics of the patients according to the RT-qPCR result from nasopharyngeal samples.

\begin{tabular}{|c|c|c|c|c|c|c|c|}
\hline & $\begin{array}{l}\text { Total } \\
n(\%)\end{array}$ & $\begin{array}{c}\text { Positive } \\
n(\%)\end{array}$ & $\begin{array}{c}\text { Negative } \\
n(\%)\end{array}$ & $p$-Value & $\begin{array}{c}\mathrm{Ct} \leq 30 \\
n(\%)\end{array}$ & $\begin{array}{c}\mathrm{Ct}>30 \\
n(\%)\end{array}$ & $p$-Value \\
\hline \multicolumn{8}{|l|}{ Sex } \\
\hline Female & 374 (55.5) & $182(54.0)$ & $192(57.0)$ & 0.438 & 79 (54.9) & 103 (53.4) & 0.786 \\
\hline Male & $300(44.5)$ & $155(46.0)$ & $145(43.0)$ & & $65(45.1)$ & $90(46.6)$ & \\
\hline \multicolumn{8}{|l|}{ Age group } \\
\hline $0-14$ years & 103 (15.3) & $48(14.2)$ & $55(16.3)$ & & $20(13.9)$ & $28(14.5)$ & \\
\hline $15-49$ years & $405(60.1)$ & 207 (61.4) & $198(58.8)$ & 0.705 & $86(59.7)$ & $121(62.7)$ & 0.749 \\
\hline$\geq 50$ years & $166(24.6)$ & $82(24.3)$ & $84(24.9)$ & & $38(26.4)$ & $44(22.8)$ & \\
\hline \multicolumn{8}{|l|}{ Chronic } \\
\hline $\begin{array}{c}\text { conditions } \\
\text { No }\end{array}$ & $499(74.0)$ & 259 (76.9) & $240(71.2)$ & 0.095 & $32(22.2)$ & $46(23.8)$ & 0.729 \\
\hline Yes & $175(26.0)$ & $78(23.1)$ & $97(28.8)$ & & $112(77.8)$ & 147 (76.2) & \\
\hline \multicolumn{8}{|l|}{ COVID-19 } \\
\hline symptoms & & & & & & & \\
\hline Yes & $333(49.4)$ & $190(56.4)$ & $143(42.4)$ & $<0.001$ & $96(66.7)$ & 94 (48.7) & 0.001 \\
\hline No & 341 (50.6) & $147(43.6)$ & $194(57.6)$ & & 48 (33.3) & $99(51.3)$ & \\
\hline \multicolumn{8}{|l|}{ Lysis buffer } \\
\hline Yes & 407 (60.4) & 208 (61.7) & 199 (59.1) & 0.479 & $82(56.9)$ & $126(65.3)$ & 0.119 \\
\hline No & 267 (39.6) & $129(38.3)$ & $138(40.9)$ & & $62(43.1)$ & $67(34.7)$ & \\
\hline
\end{tabular}


Table 1. Cont.

\begin{tabular}{|c|c|c|c|c|c|c|c|}
\hline & $\begin{array}{l}\text { Total } \\
n(\%)\end{array}$ & $\begin{array}{c}\text { Positive } \\
n(\%)\end{array}$ & $\begin{array}{c}\text { Negative } \\
n(\%)\end{array}$ & $p$-Value & $\begin{array}{c}\mathrm{Ct} \leq 30 \\
n(\%)\end{array}$ & $\begin{array}{c}\mathrm{Ct}>30 \\
n(\%)\end{array}$ & $p$-Value \\
\hline $\begin{array}{l}\text { RT-qPCR } \\
\text { manufac- } \\
\text { turer }\end{array}$ & & & & $<0.001$ & & & 0.598 \\
\hline Roche & $122(18.1)$ & $121(35.9)$ & $1(0.3)$ & & $54(37.5)$ & $67(34.7)$ & \\
\hline Seegene & $552(81.9)$ & $216(64.1)$ & 336 (99.7) & & $90(62.5)$ & $126(65.3)$ & \\
\hline Total & $674(100)$ & 337 (100) & 337 (100) & & $144(100)$ & $193(100)$ & \\
\hline
\end{tabular}

$p$-value obtained by Chi-square test of comparison RT-qPCR positive and negative patients.

\subsection{Comparison of RT-qPCR Results between Saliva and NP Samples}

Out of the 674 pairs of samples processed by RT-qPCR, 636 (94.4\%) had valid results in both NP and saliva samples. Of the $38(5.6 \%)$ patients with invalid results in saliva specimens, 13 were positive, and 25 were negative for the NP sample. Among the 636 pairs of samples with a valid result, $477(75.0 \%)$ showed concordant results between NP and saliva (168 positives and 309 negatives) and $159(25.0 \%)$ showed discordant results (156 negatives in saliva and positive in NP; and three saliva positive and NP negative) (Figure 1).

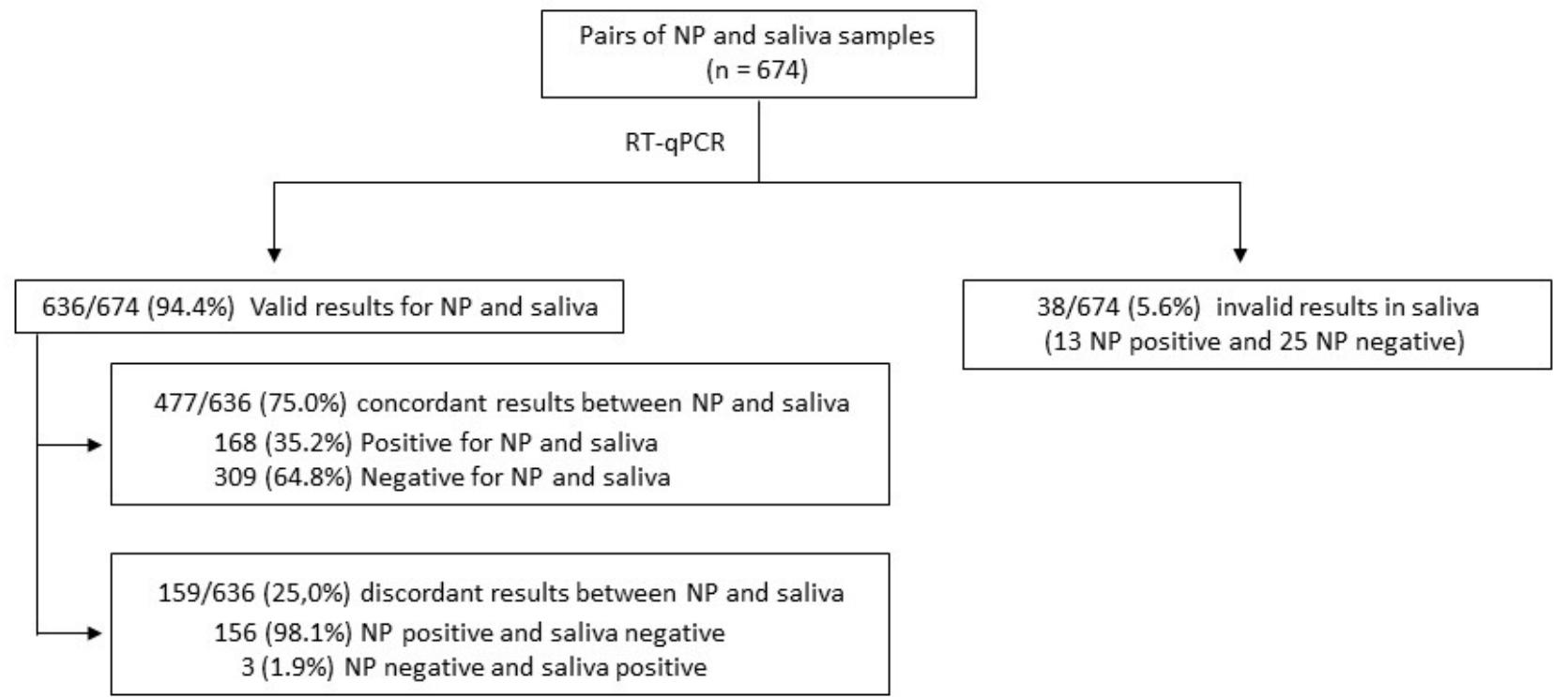

Figure 1. Summary of RT-qPCR results in saliva and nasopharyngeal (NP) samples.

When comparing the $\mathrm{Ct}$ values for patients with an RT-qPCR positive result in both samples, the median Ct value of NP samples $(23.63 \pm 6.96)$ was lower than that of the saliva specimens $(28.60 \pm 5.94)$. The difference between means was statistically significant $(4.96 \pm 5.68 ; p<0.001)$.

\subsection{Sensitivity and Specificity of Saliva Samples}

One out of two NP positives was detected with saliva samples. Excluding invalid results, the sensitivity and specificity for saliva samples were $51.9 \%$ (95\% CI: $46.3 \%-57.4 \%$ ) and $99.1 \%$ (95\% CI: $97.4 \%-99.8 \%$ ), respectively. Including invalid results in saliva samples, the sensitivity values were similar, indicating that these data exclusions would not significantly influence the outcome (Table 2). The concordance rate between the two samples was $75 \%(\mathrm{~K}=0.50$; $95 \%$ CI: 0.45-0.56) (Table 2).

Virus detection (sensitivity) in samples with $\mathrm{Ct} \leq 30$ was $91.6 \%$ (95\% CI: 85.7\%-95.6\%); however, in the samples with Ct $>30$ it was $20.0 \%(95 \%$ CI: $14.4 \%-26.6 \%)(p<0.001)$. 
Table 2. Sensitivity (according to Ct and total), specificity, and kappa index of the saliva samples as compared with the result of nasopharyngeal samples.

\begin{tabular}{|c|c|c|c|c|c|c|}
\hline & \multicolumn{4}{|c|}{ Sensitivity, \% (95\% CI) } & \multirow{2}{*}{$\begin{array}{c}\text { Specificity, \% } \\
(95 \% \text { CI })\end{array}$} & \multirow{2}{*}{$\begin{array}{c}\text { Kappa Index } \\
(95 \% \mathrm{CI})\end{array}$} \\
\hline & All Samples & $\begin{array}{l}\text { All Valid } \\
\text { Results }\end{array}$ & $\mathrm{Ct} \leq \mathbf{3 0}$ & $\mathrm{Ct}>30$ & & \\
\hline Total & $\begin{array}{c}49.9 \\
(44.4-55.3)\end{array}$ & $\begin{array}{c}51.9 \\
(46.3-57.4)\end{array}$ & $\begin{array}{c}91.6 \\
(86.7-96.5)\end{array}$ & $\begin{array}{c}20.4 \\
(14.3-26.6)\end{array}$ & $\begin{array}{c}99.1 \\
(97.4-99.8)\end{array}$ & $\begin{array}{c}0.50 \\
(0.45-0.56)\end{array}$ \\
\hline \multicolumn{7}{|l|}{$\begin{array}{c}\text { Age group } \\
\text { (years) }\end{array}$} \\
\hline Children (0-14) & $\begin{array}{c}56.3 \\
(41.2-71.3)\end{array}$ & $\begin{array}{c}60.0 \\
(44.6-75.4)\end{array}$ & $\begin{array}{c}100 \\
(83.3-100)\end{array}$ & $\begin{array}{c}28.0 \\
(8.4-47.6)\end{array}$ & $\begin{array}{c}100 \\
(93.5-100)\end{array}$ & $\begin{array}{c}0.62 \\
(0.47-0.76)\end{array}$ \\
\hline Adults (>14) & $\begin{array}{c}48.8 \\
(42.9-54.7)\end{array}$ & $\begin{array}{c}50.5 \\
(44.5-56.6)\end{array}$ & $\begin{array}{c}90.2 \\
(84.6-95.9)\end{array}$ & $\begin{array}{c}19.2 \\
(12.7-25.7)\end{array}$ & $\begin{array}{c}98.9 \\
(96.9-99.8)\end{array}$ & $\begin{array}{c}0.49 \\
(0.42-0.55)\end{array}$ \\
\hline \multicolumn{7}{|l|}{$\begin{array}{l}\text { Chronic } \\
\text { conditions }\end{array}$} \\
\hline No & $\begin{array}{c}49.8 \\
(43.7-55.9)\end{array}$ & $\begin{array}{c}51.8 \\
(45.6-58.0)\end{array}$ & $\begin{array}{c}91.9 \\
(85.6-96.0)\end{array}$ & $\begin{array}{c}19.6 \\
(13.6-26.8)\end{array}$ & $\begin{array}{c}100 \\
(93.7-100)\end{array}$ & $\begin{array}{c}0.50 \\
(0.44-0.57)\end{array}$ \\
\hline Yes & $\begin{array}{c}50.0 \\
(39.0-61.0)\end{array}$ & $\begin{array}{c}52.0 \\
(40.7-63.1)\end{array}$ & $\begin{array}{c}90.6 \\
(76.6-97.6)\end{array}$ & $\begin{array}{c}23.3 \\
(12.5-37.6)\end{array}$ & $\begin{array}{c}96.6 \\
(91.0-99.1)\end{array}$ & $\begin{array}{c}0.50 \\
(0.38-0.63)\end{array}$ \\
\hline \multicolumn{7}{|l|}{$\begin{array}{l}\text { COVID-19 } \\
\text { symptoms }\end{array}$} \\
\hline Yes & $\begin{array}{c}55.3 \\
(47.9-62.6)\end{array}$ & $\begin{array}{c}56.5 \\
(49.1-63.8)\end{array}$ & $\begin{array}{c}94.7 \\
(88.1-98.3)\end{array}$ & $\begin{array}{c}16.5 \\
(8.3-24.7)\end{array}$ & $\begin{array}{c}100 \\
(97.5-100)\end{array}$ & $\begin{array}{c}0.52 \\
(0.44-0.60)\end{array}$ \\
\hline No & $\begin{array}{c}42.9 \\
(34.5-51.2)\end{array}$ & $\begin{array}{c}45.7 \\
(37.0-54.3)\end{array}$ & $\begin{array}{c}85.4 \\
(74.4-96.4)\end{array}$ & $\begin{array}{c}24.4 \\
(15.0-33.9)\end{array}$ & $\begin{array}{c}98.5 \\
(95.5-99.7)\end{array}$ & $\begin{array}{c}0.47 \\
(0.38-0.56)\end{array}$ \\
\hline \multicolumn{7}{|l|}{ Processing } \\
\hline $\begin{array}{l}\text { With lysis } \\
\text { buffer }\end{array}$ & $\begin{array}{c}46.2 \\
(39.1-53.2)\end{array}$ & $\begin{array}{c}49.2 \\
(42.0-56.5)\end{array}$ & $\begin{array}{c}92.6 \\
(86.3-98.9)\end{array}$ & $\begin{array}{c}18.4 \\
(10.9-26.0)\end{array}$ & $\begin{array}{c}99.0 \\
(96.4-99.9)\end{array}$ & $\begin{array}{c}0.47 \\
(0.39-0.54)\end{array}$ \\
\hline $\begin{array}{l}\text { Without lysis } \\
\text { buffer }\end{array}$ & $\begin{array}{c}55.8 \\
(46.9-64.8)\end{array}$ & $\begin{array}{c}55.8 \\
(46.9-64.8)\end{array}$ & $\begin{array}{c}90.3 \\
(82.2-98.5)\end{array}$ & $\begin{array}{c}23.9 \\
(12.9-34.8)\end{array}$ & $\begin{array}{c}99.3 \\
(96.0-100)\end{array}$ & $\begin{array}{c}0.56 \\
(0.47-0.65)\end{array}$ \\
\hline
\end{tabular}

$\mathrm{CI}=$ confidence interval calculated by the exact method.

\subsection{Results under Different Conditions}

\subsubsection{Age Groups and Chronic Conditions}

Among the children, 60\% (95\% CI: 44.6\%-75.4\%) of the NP positive patients were also detected in the saliva sample, but sensitivity increased up to $100 \%$ (95\% CI: $83.3 \%$ $100 \%$ ) in samples with $\mathrm{Ct} \leq 30$, while only $28.0 \%$ (95\% CI: $8.4 \%-47.6 \%)$ in those with $\mathrm{Ct}>30(p<0.001)$. In adults, 50.5\% (95\% CI: 44.5\%-56.6\%) of all NP positive patients were also detected in the saliva sample, with a sensibility of $90.2 \%$ (95\% CI: $84.6 \%-95.9 \%)$, and $19.2 \%(95 \% \mathrm{CI}: 12.7 \%-25.7 \%)$ in those with $\mathrm{Ct} \leq 30$ and $\mathrm{Ct}>30$, respectively $(p<0.001)$. Presence of chronic conditions did not affect the results from saliva samples (Table 2).

\subsubsection{COVID-19 Symptoms}

In symptomatic patients, saliva samples detected 56.5\% (95\% CI: 49.1\%-63.8\%) of NP positive patients, and sensibility was $94.7 \%$ (95\% CI: $88.1 \%-98.3 \%$ ) in those with $\mathrm{Ct} \leq 30$, but only $16.5 \%(95 \% \mathrm{CI}: 8.3 \%-24.7 \%)$ of those with $\mathrm{Ct}>30(p<0.001)$. In asymptomatic patients, saliva samples had a sensitivity of $45.7 \%$ (95\% CI: 37.0\%-54.3\%) overall, 85.4\% (95\% CI: 74.4\%-96.4\%) in those with Ct $\leq 30$, and 24.4\% (95\% CI: 15.0\%-33.9\%) in those with $\mathrm{Ct}>30$. Sensitivity was not statistically significantly different by presence of symptoms $(p=0.054)$ (Table 2).

It should be noted that three cases were detected in NP negative patients with a saliva positive sample. All of them were asymptomatic adult patients with $\mathrm{Ct}>35$. 


\subsubsection{Processing with/without Lysis Buffer}

Four hundred and seven $(60.4 \%)$ saliva samples were diluted in lysis buffer, and 267 (39.6\%) were processed directly. In the first group, 270 (66.3\%) samples showed concordant results between NP and saliva; among those samples processed directly (without lysis buffer), 207 (77.5\%) presented concordant results between NP and saliva ( $p=0.125)$.

There were no significant differences in sensitivity and specificity by age, COVID-19 symptoms, and the saliva sample processing (Table 2).

\section{Discussion}

Nowadays, a proper diagnosis of COVID-19 is crucial to control the pandemic that is ravaging the world. NP and OP swabs are the recommended specimens to be analyzed by RT-qPCR. However, these samples have several limitations such as the invasive extraction procedure and healthcare professionals' skills to perform the method. For this reason, it is essential to find a new clinical sample type that is reliable, less invasive, and cost-effective for the detection of SARS-CoV-2 [5,7].

Saliva specimens may be a good alternative for the detection of SARS-CoV-2. The US Food and Drug Administration has approved the use of these samples to diagnose COVID$19[7,14]$. Several studies compared RT-qPCR test results from saliva samples and those from NP or OP samples in patients with suspected or confirmed COVID-19 [5,8,9,15,16]. However, doubts remain regarding the validity of saliva sample results, as the sensitivity may be significantly lower than the NP sample considered as the gold standard.

In the present study, 674 pairs of NP and saliva samples were analyzed by RT-qPCR to validate the utility of saliva samples to diagnose SARS-CoV-2. Considering the NP samples as the gold standard, the saliva samples showed very high specificity $(99.1 \%)$, which means that the saliva sample is very useful for detecting true negatives since it correctly classifies healthy patients. However, the sensibility was modest (51.9\%), so the saliva sample showed a limited capacity to detect true positives. Other studies have also described this fact, with sensitivities for the saliva sample ranging from $31 \%$ to $100 \%[5,8,9,15,17]$. However, it is remarkable that our research is the one with the most significant sample size analyzed. Besides, we found a moderate concordance level among NP and saliva samples $(\kappa=0.50)$ [16]. This kappa index value obtained in our study is higher than the obtained in a previous study [5], but Pasomsub et al. reported a better kappa value in a study conducted with 200 samples [18]. The variability in results among studies may be due to the sample size, the study's design, the methodology used, the stage of the patients, and the prevalence of COVID-19 in each region. The present study had higher statistical power than previous studies due to the increased sample size and the inclusion of a similar number of RT-qPCR positive and negative patients. Other advantages were that pairs of samples were obtained simultaneously, and results were evaluated under different conditions.

The RT-qPCR performance on the saliva sample offered high sensitivity for cases with a $\mathrm{Ct} \leq 30$ and low for those with $\mathrm{Ct}>30$, regardless of the age, chronic conditions, and presence of symptoms. Despite the discrepancies in the importance of $\mathrm{Ct}$ values, a priori the risk of transmission is greater in patients with low $\mathrm{Ct}$. The viral load is high and infectivity could also be higher, particularly for asymptomatic patients or those with mild symptoms [13]. The percentage of false negative results would decrease with repeated samples since high initial cycle times are not durable over time. Although high $\mathrm{Ct}$ values can last over time once the disease has passed, there are doubts about these individuals infectivity, so the loss of these cases would be less important for epidemiological control and prevention.

Recent studies have demonstrated the utility of saliva samples in asymptomatic patients to detect SARS-CoV-2 $[9,17,19]$. We analyzed 341 samples from asymptomatic and 333 from symptomatic patients, and no significant differences were observed in the sensitivity, $45.7 \%$ and $56.5 \%$, respectively. 
We confirmed three cases (1.9\% out of confirmed patients) in which the virus was detected in saliva but not in the NP sample. Considering the NP swabs results as the gold standard, and making an exception, these samples could be probably associated with type II error, because of the difficulties in the correct NP swab collection. Other studies have reported similar findings $[5,15,17-21]$.

One of the limitations of this study was the collection of the saliva sample. Although patients were previously informed of the procedure for collecting the saliva samples, if the patient did not follow the instructions, this may affect the results. Different studies suggest that the collection of the sample can influence the result [8,10,20-22]. Saliva can be collected directly from the salivary gland canal with buccal or lingual swabs, pouring the saliva that accumulates in the oral cavity into a sterile container or obtaining saliva from the posterior OP [22]. Some authors suggest that the use of saliva from the OP provides higher sensitivity, but if sputum is collected instead of saliva, sensitivity results may be biased, mainly in entirely asymptomatic patients $[8,10,22,23]$.

Regarding the time of collection of the saliva sample, Hung et al. hypothesized that collecting the first hour of the morning could increase the sensitivity [10]. In our case, the saliva samples were collected following the oral cavity accumulation protocol and were not collected from the first hour of the morning, and specimens were frozen at $-80{ }^{\circ} \mathrm{C}$ until its processing. The present study included NP samples that were tested using two different platforms. However, both methods detect the E SARS-CoV-2 gene and are validated for diagnosis. Both platforms are widely accepted as the gold standard for SARS-CoV-2 detection.

Several protocols suggest the use of stabilizing buffers and additives for preserving saliva specimens, and others consider that these buffers are not necessary and may inhibit RT-qPCR [11]. We have evaluated and compared the results of adding or not adding lysis buffer. No statistically significant differences were observed, so the sample treatment with lysis buffer does not seem to influence the results.

\section{Conclusions}

Based on our results, there are several key points to consider for getting definitive conclusions. The procedure of collecting the saliva sample is one of the main points to obtain satisfactory results. Due to its low sensitivity, the saliva sample should not replace the NP sample in all situations. For example, it does not seem suitable for hospital diagnosis, screening in patients who are going to receive health care for other reasons, and high-risk contacts. However, the saliva sample could be a useful alternative to the NP samples in massive screening studies (schools, workplaces, geographical area, etc.) when the availability of trained professionals for sampling or personal protection equipment is limited. Finally, it is essential to highlight that saliva sample collection is a less invasive procedure that can contribute to the patient comfort and may not need health care professional assistance and the subsequent spending of their protective equipment.

Supplementary Materials: The following are available online at https:/ / www.mdpi.com/2077-038 3/10/2/299/s1. Protocol S1: Saliva sample collection protocol for SARS-CoV-2 RT-qPCR.

Author Contributions: A.N., C.E., I.R.-R. and J.C. designed the study and coordinated the activities. C.T.-S., M.J.G., I.R.-R. and J.C. undertook the statistical analysis. A.N., C.E., C.T.-S. and M.A. were responsible for the virology analysis and interpretation of laboratory results. C.T.-S. wrote the draft manuscript. All authors have read and agreed to the published version of the manuscript.

Funding: This work was supported by the Horizon 2020 program of the European Commission (I-MOVE-COVID-19, grant agreement No 101003673) and the Carlos III Institute of Health with the European Regional Development Fund (COV20/00542 and INT19/00028).

Institutional Review Board Statement: The Ethical Committee for Clinical Research of Navarra approved the study protocol, PI_2020/96.

Informed Consent Statement: All samples were obtained with the verbal consent of participants. 
Data Availability Statement: All available information is included in this article.

Acknowledgments: The authors acknowledge Sergio Santana Domínguez, Leire Leache Alegría, Marta Gutiérrez Valencia, Luis Carlos Saíz and Juan Erviti López of the Navarra Health Service; as well as, Ana Ariztegui Echenique and Pilar Sola Sara of the Primary Care Management. Furthermore, we would like to acknowledge César Ignacio Fernández Lázaro for the improvement of the English of the draft manuscript.

Conflicts of Interest: The authors declare no conflict of interest.

\section{References}

1. Lahner, E.; Dilaghi, E.; Prestigiacomo, C.; Alessio, G.; Marcellini, L.; Simmaco, M.; Santino, I.; Orsi, G.B.; Anibaldi, P.; Marcolongo, A.; et al. Prevalence of Sars-Cov-2 Infection in Health Workers (HWs) and Diagnostic Test Performance: The Experience of a Teaching Hospital in Central Italy. Int. J. Environ. Res. Public Health. 2020, 17, 4417. [CrossRef] [PubMed]

2. Słomka, A.; Kowalewski, M.; Żekanowska, E. Coronavirus Disease 2019 (COVID-19): A Short Review on Hematological Manifestations. Pathogens 2020, 9, 493. [CrossRef] [PubMed]

3. Singh, V.K.; Mishra, A.; Singh, S.; Kumar, P.; Singh, M.; Jagannath, C.; Khan, A. Emerging Prevention and Treatment Strategies to Control COVID-19. Pathogens 2020, 9, 501. [CrossRef] [PubMed]

4. Azzi, L.; Carcano, G.; Gianfagna, F.; Grossi, P.; Gasperina, D.D.; Genoni, A.; Fasano, M.; Sessa, F.; Tettamanti, L.; Carinci, F.; et al. Saliva is a Reliable Tool to Detect SARS-CoV-2. J. Infection 2020, 81, e45-e50. [CrossRef]

5. Rao, M.; Rashid, F.A.; Sabri, F.S.A.H.; Jamil, N.N.; Zain, R.; Hashim, R.; Amran, F.; Kok, H.T.; Samad, A.A.; Ahmad, N. Comparing Nasopharyngeal Swab and Early Morning Saliva for the Identification of Severe Acute Respiratory Syndrome Coronavirus 2 (SARS-CoV-2). Clin. Infect. Dis. 2020, 1156. [CrossRef]

6. World Health Organization. Clinical Management of Severe Acute Respiratory Infection (SARI) when COVID-19 Disease is Suspected. Interim guidance. Pediatr. I Med. Rodz. 2020, 16, 9-26. [CrossRef]

7. Cerón, J.; Lamy, E.; Martínez-Subiela, S.; López-Jornet, P.; Eckersall, P.D.; Eckersall, P.D.; Asta, T. Use of Saliva for Diagnosis and Monitoring the SARS-CoV-2: A General Perspective. J. Clin. Med. 2020, 9, 1491. [CrossRef]

8. To, K.K.; Yip, C.C.; Lai, C.Y.; Wong, C.K.; Ho, D.T.; Pang, P.K.; Ng, A.C.; Leung, K.-H.; Poon, R.W.; Chan, K.-H.; et al. Saliva as a Diagnostic Specimen for Testing Respiratory Virus by a Point-of-Care Molecular Assay: A Diagnostic Validity Study. Clin. Microbiol. Infect. 2019, 25, 372-378. [CrossRef]

9. Wyllie, A.; Fournier, J.; Casanovas-Massana, A.; Campbell, M.; Tokuyama, M.; Vijayakumar, P.; Warren, J.L.; Geng, B.; Muenker, M.C.; Moore, A.J.; et al. Saliva or Nasopharyngeal Swab Specimens for Detection of SARS-CoV-2. N. Engl. J. Med. 2020, 383, 1283-1286. [CrossRef]

10. Hung, D.L.-L.; Li, X.; Chiu, K.H.-Y.; Yip, C.C.-Y.; To, K.K.-W.; Chan, J.F.-W.; Sridhar, S.; Chung, T.W.-H.; Lung, K.-C.; Liu, R.W.-T.; et al. Early-Morning vs Spot Posterior Oropharyngeal Saliva for Diagnosis of SARS-CoV-2 Infection: Implication of Timing of Specimen Collection for Community-Wide Screening. Open Forum Infect. Dis. 2020, 7, ofaa210. [CrossRef]

11. Vogels, C.B.; Brackney, D.; Wang, J.; Kalinich, C.C.; Ott, I.M.; Kudo, E.; Lu, P.; Venkataraman, A.; Tokuyama, M.; Moore, A.J.; et al. SalivaDirect: Simple and Sensitive Molecular Diagnostic Test for SARS-CoV-2 Surveillance. MedRxiv 2020. [CrossRef]

12. Jaafar, R.; Aherfi, S.; Wurtz, N.; Grimaldier, C.; Van Hoang, T.; Colson, P.; Raoult, D.; La Scola, B. Correlation Between 3790 Quantitative Polymerase Chain Reaction-Positives Samples and Positive Cell Cultures, Including 1941 Severe Acute Respiratory Syndrome Coronavirus 2 Isolates. Clin. Infect. Dis. 2020, 1491. [CrossRef] [PubMed]

13. Singanayagam, A.; Patel, M.; Charlett, A.; Bernal, J.L.; Saliba, V.; Ellis, J.; Ladhani, S.; Zambon, M.; Gopal, R. Duration of infectiousness and correlation with RT-PCR cycle threshold values in cases of COVID-19, England, January to May 2020. Eurosurveillance 2020, 25, 2001483. [CrossRef] [PubMed]

14. First Saliva Test for COVID-19 Approveed for Emergency use by FDA I The Scientist Magazine®. Available online: https: / / www.the-scientist.com/news-opinion/first-saliva-test-for-covid-19-approved-for-emergency-use-by-fda-67416 (accessed on 6 August 2020).

15. Iwasaki, S.; Fujisawa, S.; Nakakubo, S.; Kamada, K.; Yamashita, Y.; Fukumoto, T.; Sato, K.; Oguri, S.; Taki, K.; Senjo, H.; et al. Comparison of SARS-CoV-2 Detection in Nasopharyngeal Swab and Saliva. J. Infect. 2020, 81, e145-e147. [CrossRef]

16. Landis, J.R.; Koch, G.G. The Measurement of Observer Agreement for Categorical Data. Biometrics 1977, 33, 159-174. [CrossRef]

17. Jamal, A.J.; Mozafarihashjin, M.; Coomes, E.; Powis, J.; Li, A.X.; Paterson, A.; Anceva-Sami, S.; Barati, S.; Crowl, G.; Faheem, A.; et al. Sensitivity of Nasopharyngeal Swabs and Saliva for the Detection of Severe Acute Respiratory Syndrome Coronavirus 2. Clin. Infect. Dis. 2020, 25, ciaa848. [CrossRef]

18. Pasomsub, E.; Watcharananan, S.; Boonyawat, K.; Janchompoo, P.; Wongtabtim, G.; Suksuwan, W.; Sungkanuparph, S.; Phuphuakrat, A. Saliva Sample as a Non-invasive Specimen for the Diagnosis of Coronavirus Disease 2019: A Cross-Sectional Study. Clin. Microbiol. Infect. 2020, 15, 1198. [CrossRef]

19. Williams, E.; Bond, K.; Zhang, B.; Putland, M.; Williamson, D.A. Saliva as a Noninvasive Specimen for Detection of SARS-CoV-2. J. Clin. Microbiol. 2020, 58. [CrossRef]

20. Ye, G.; Li, Y.; Lu, M.; Chen, S.; Luo, Y.; Wang, S.; Wang, Y.; Wang, X. Experience of Different Upper Respiratory Tract Sampling Strategies for Detection of COVID-19. J. Hosp. Infect. 2020, 105, 1-2. [CrossRef] 
21. Zhu, J.; Guo, J.; Xu, Y.; Chen, X. Viral Dynamics of SARS-CoV-2 in Saliva from Infected Patients. J. Infect. 2020, 81, e48-e50. [CrossRef]

22. To, K.K.; Tsang, O.T.-Y.; Yip, C.C.-Y.; Chan, K.-H.; Wu, T.-C.; Chan, J.M.-C.; Leung, W.-S.; Chik, T.S.-H.; Choi, C.Y.-C.; Kandamby, D.H.; et al. Consistent Detection of 2019 Novel Coronavirus in Saliva. Clin. Infect. Dis. 2020, 71, 841-843. [CrossRef] [PubMed]

23. Xu, R.; Cui, B.; Duan, X.; Zhang, P.; Zhou, X.; Yuan, Q. Saliva: Potential Diagnostic Value and Transmission of 2019-nCoV. Int. J. Oral Sci. 2020, 12, 11. [CrossRef] [PubMed] 